УДК 004.032.26

Е. Е. Федоров ${ }^{1}$, д.т.н., доиент, профессор, e-mail: fedorovee75@ukr.net

И. В. Ярош ${ }^{2}$, стариий преподаватель, e-mail: iryna.yarosh@donntu.edu.ua

Т. А. Черняк ${ }^{2}$, ассистент,

e-mail: tetiana.cherniak@donntu.edu.ua

${ }^{1}$ Черкасский государственный технологический университет

б-р Шевченко, 460, г. Черкассы, 18006, Украина

${ }^{2}$ Донецкий национальный технический университет

пл. Шибанкова, 2, г. Покровск, 85300, Украина

\title{
МЕТОД ПАКЕТНОГО ОБУЧЕНИЯ НЕЙРОСЕТИ \\ С ЗАДЕРЖКОЙ ВО ВХОДНОМ СЛОЕ ДЛЯ КОМПЛЕКСНОЙ ДИАГНОСТИКИ СОСТОЯНИЯ ВЕНТИЛЯТОРНОЙ УСТАНОВКИ ГЛАВНОГО ПРОВЕТРИВАНИЯ
}

В статье рассмотрены и представлены данные выполненного анализа имеюшихся методов для осуществления диагностики состояния вентиляторной установки главного проветривания на горнопромышленном предприятии (шахте). Выполненные исследования направлены на снижение вероятности возникновения ошибок диагностики. Достоинства и недостатки указанных существующих методов учтены при разработке и реализации нейросетевого метода диагностики вентиляторной установки главного проветривания. Проведенные эксперименть позволили создать архитектуру нейронной сети с определенной задержкой во входном слое, которая послужила основой для разработки приведенного метода. Возможность ускоренного обучения предоставляется за счет предложенного в работе пакетного режима. Эффективность метода была оценена в результате множественных исследований, которые отражают работоспособность сети и архитектуры. Предложенная в работе нейронная сеть позволяет получить минимум отклонений при диагностике.

Ключевые слова: комплексная диагностика, вентиляторная установка главного проветривания, искусственная нейронная сеть, производственная безопасность, пакетный режим обучения.

Введение. Повышение безопасности на производстве в сфере горной промышленности относится к наиболее актуальным вопросам сегодняшнего дня. Шахтное оборудование находится в большинстве своем в аварийном состоянии по причине стремительно растущей доли исчерпания его эксплуатационного ресурса. Из-за несвоевременного проведения комплексной диагностики, которая должна быть выполнена по нескольким параметрам с применением существующих компьютерных систем диагностики [1-6], меры, используемые для предотвращения или сокращения вероятности неблагоприятных последствий, могут предприниматься с опозданием или вовсе не учитывать все факторы. Вентиляторные установки главного проветривания (ВУГП) это одни из ключевых сооружений и оборудований на горнопромышленном предприятии, ведущем подземную добычу полезного ископаемого (на шахте). С их помощью обеспечи- вается нормальная жизнедеятельность шахтного персонала, что обуславливает необходимость создания методов интеллектуальной диагностики вентиляторной установки главного проветривания.

Цель исследования заключается в разработке метода исследования процесса изменения работоспособности ВУГП.

Задачи исследования, результат постановки и решения которых приведет к достижению указанной цели исследования:

- выполнить анализ имеющихся методов диагностики;

- провести разработку искусственной нейронной сети диагностики;

- установить структурные элементы модели искусственной нейронной сети;

- определить параметры оценивания эффективности нейронной сетевой модели диагностики; 
- выполнить обучение модели искусственной нейронной сети;

- провести численные исследования.

Изложение основного материала. В качестве основного материала будут приведены результаты разработки и выполненных исследований.

Сегодня существует целый ряд подходов, используемых для вибрационной диагностики. Из них выделяют такие методы вычисления, как:

- эксцесс;

- среднеквадратичное значение;

- пик-фактор;

- спектр огибающей.

Сравнительная характеристика этих методов присутствует в работах [1-6]. Тем не менее, минимальная вероятность ошибки, в случае их применения для диагностики состояния ВУГП, равна 0.05 .

Диагностика, выполненная с помощью искусственных нейронных сетей, позволяет говорить о ряде неоспоримых преимуществ, среди которых следует выделить следующие моменты:

- исследование взаимосвязи между факторами на существующих моделях;

- распределение факторов не предполагает выдвижения каких-либо предположений;

- наличие исходных данных о фактоpax не является обязательным условием;

- допустимы зашумленность, неполнота и сильная корреляция первичной информации;

- системы с высокой степенью нелинейности могут быть проанализированы;

- достаточно высокая степень адаптивности и скорость разработки модели;

- выполнение анализа систем, которые содержат множество факторов;

- отсутствие потребности и необходимости в полном пересмотре всех допустимых моделей;

- вероятность проведения анализа систем, имеющих неоднородные факторы.

В качестве метода диагностики в данной статье выбран нейросетевой способ.

Наиболее распространенными искусственными нейронными сетями являются следующие [17]:

- многослойный персептрон (MLP) [910], представляющий собой статическую нерекуррентную многослойную сеть;
- рекуррентный многослойный персептрон (RMLP) [9-10], который выступает в качестве рекуррентной многослойной сети, построенной на базе MLP;

- нейросеть с радиально-базисными функциями (RBFNN) [11-12], которая представляет статическую нерекуррентную двухслойную сеть, разработанную на базе MLP;

- нейросеть нелинейной авторегрессии (NARNN) [13-14], которая отражает динамическую нерекуррентную двухслойную сеть, построенную на базе MLP;

- нейросеть нелинейной авторегрессии-скользящего среднего (NARMANN) [1517], представляющая собой рекуррентную двухслойную сеть, выполненную на базе MLP;

- авторская нейросеть - динамическая нерекуррентная двухслойная сеть, которая построена на базе MLP.

Результаты анализа и сравнительной характеристики искусственных нейронных сетей отражены в табл. 1.

Авторская нейросеть выбрана для решения задач диагностики ввиду присутствия в ней всех трех параметров.

На рис. 1 представлена структура авторской нейросети с задержкой во времени во входном слое.

Формализованное представление:

$$
\begin{gathered}
y^{(0)}(n)=x_{1} \cdot \\
y_{j}^{(1)}(n)=f^{(1)}\left(s_{j}^{(1)}(n)\right), \quad j \in \overline{1, N^{(1)}}, \\
s_{j}^{(1)}(n)=b_{j}^{(1)}+\sum_{t=0}^{M^{(0)}} \sum_{i=1}^{N^{(0)}} w_{i t j}^{(1)} y_{i}^{(0)}(n-t), \\
y_{j}^{(2)}(n)=f^{(2)}\left(s_{j}^{(2)}(n)\right), j \in \overline{1, N^{(2)}}, \\
s_{j}^{(2)}(n)=b_{j}^{(2)}+\sum_{i=1}^{N_{i j}^{(1)}} w_{i j}^{(2)} y_{i}^{(1)}(n) .
\end{gathered}
$$

где $\mathrm{N}^{(\mathrm{k})}$ - число нейронов в $\mathrm{k}-\mathrm{M}$ слое, $\mathrm{b}_{\mathrm{j}}^{(\mathrm{k})}$ - порог для j-го нейрона k-го слоя, $\mathrm{w}_{\mathrm{ltj}}^{(1)}-$ вес связи от і -го нейрона 0-го слоя к j-му нейрону 1-го слоя, $w_{\mathrm{lj}}^{(2)}-$ вес связи от $i$-го нейрона 1-го слоя к ј-му нейрону 2-го слоя, $y_{j}^{(k)}(n)-$ выход j-го нейрона в k -м слое, $\mathrm{f}^{(\mathrm{k})}$ - функция активации нейронов $\mathrm{k}-$ го слоя. 
Таблица 1

\begin{tabular}{|c|c|c|c|c|c|c|}
\hline Критерий & $\stackrel{g}{\Sigma}$ & $\sum_{2}$ & $\frac{Z}{\text { Z⿱ }}$ & $\begin{array}{l}\text { Z } \\
\text { Z } \\
\text { Z }\end{array}$ & $\frac{3}{3}$ & 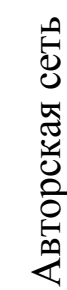 \\
\hline Использование нескольких признаков & + & + & + & - & - & + \\
\hline Задержка во входном слое & - & - & - & + & + & + \\
\hline Высока степень параллелизма & + & - & + & + & - & + \\
\hline
\end{tabular}

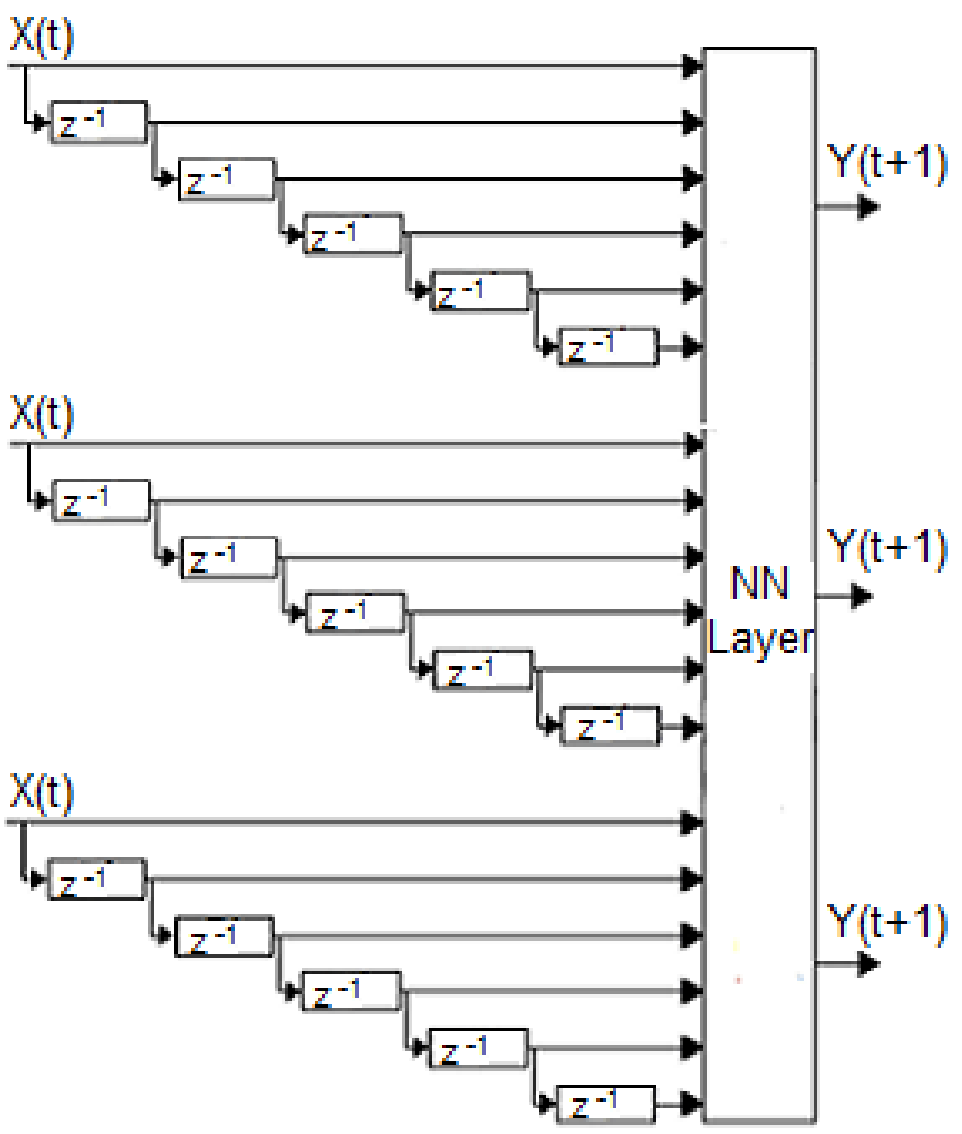

Рис. 1. Структура авторской нейросети с задержкой во времени во входном слое

Ряд проведенных экспериментальных исследований (рис. 2) был выполнен с целью выявления структуры модели авторской нейросети (определения числа скрытых нейронов).

Входными данными, используемыми для обучения предложенной авторской нейронной сети, послужила выборка значений трех показателей, основанных на показателях вибрационного сигнала, - эксцесса, среднеквадратичного значения, пик-фактора.

Для выходных данных взяты соответствующие им состояния - пригоден (дальнейшая эксплуатация возможна без ограничения по срокам), непригоден (длительная непрерывная эксплуатация невозможна), требует срочного ремонта (эксплуатация невозможна вообще). 
Минимальная

среднеквадратичная ошибка диагностики стала параметром для выбора структуры сетевой модели.

На рис. 2 представлены результаты выполнения ряда экспериментов в виде гистограммы.
Данная гистограмма показывает зависимости значений среднеквадратичной ошибки от числа нейронов в скрытом слое.

Рис. 2 демонстрирует определенную зависимость - снижение значения ошибки при увеличении числа скрытых нейронов.

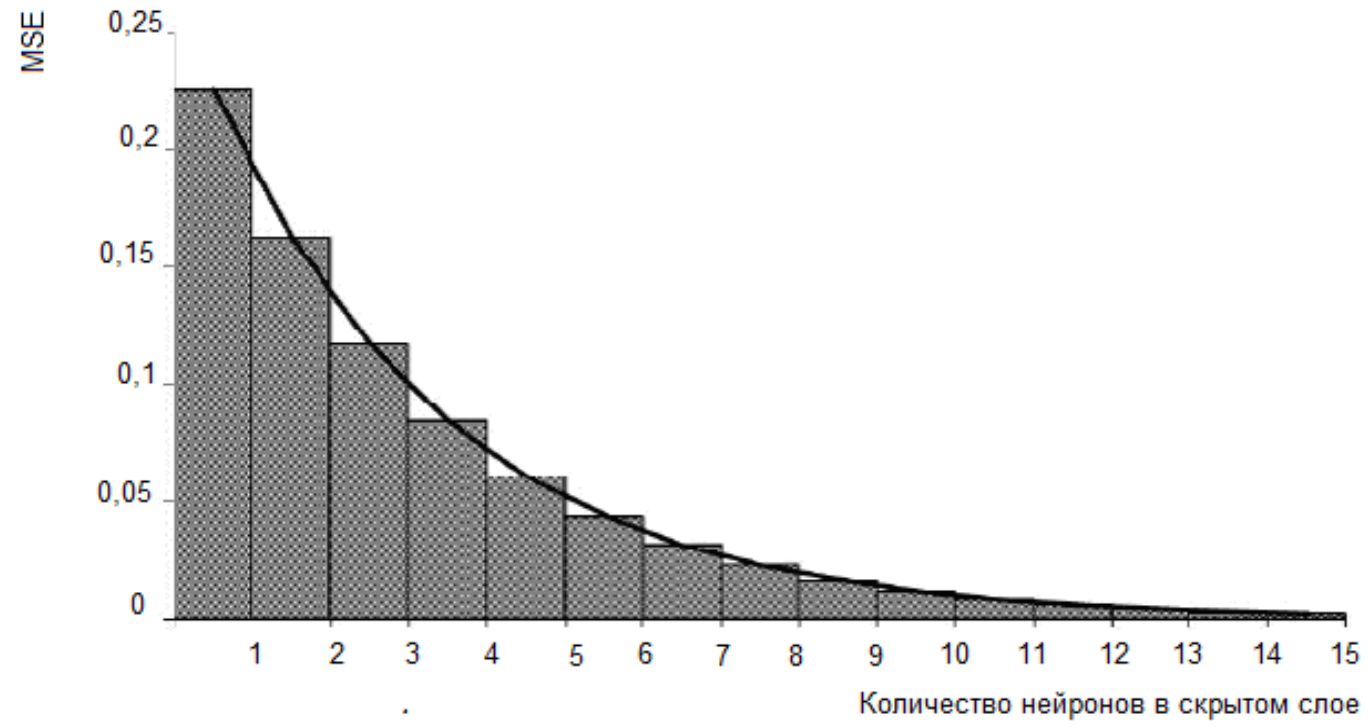

Рис. 2. Гистограмма зависимости значении среднеквадратичной ошибки от числа нейронов в скрытом слое

Оптимальное количество срытых нейронов для диагностики состояния вентилятора шахты - 10. Это связано с несущественными изменениями значений ошибки при последующем увеличении числа скрытых нейронов.

Обучение модели предложенной (авторской) нейронной сети в работе проводилось на основе критерия адекватности модели, заключающегося в выборе значений параметров функции $\mathrm{W}=\left\{\mathrm{w}_{\mathrm{itj}}^{(1)}, \mathrm{w}_{\mathrm{ij}}^{(2)}\right\}$, содержащих минимальный показатель среднеквадратичной ошибки (разности выхода по модели и желаемого выхода):

$$
\mathrm{F}=\frac{1}{\mathrm{P}} \sum_{\mathrm{p}=1}^{\mathrm{P}}\left(\mathrm{y}_{\mathrm{p}}-\mathrm{d}_{\mathrm{p}}\right)^{2} \rightarrow \min _{\mathrm{W}}
$$

Критерий (1) является ключевым для обучения модели авторской нейросети. С этой целью в статье было предложено использовать пакетный режим.

В его основу входят следующие перечисленные параметры:

1. Номер итерации обучения $\mathrm{n}=1$, инициализация смещений (порогов) $\mathrm{b}_{\mathrm{j}}^{(1)}(\mathrm{n})$, $\mathrm{j} \in \overline{1, \mathrm{~N}^{(1)}}, \quad \mathrm{b}_{\mathrm{j}}^{(2)}(\mathrm{n}), \quad \mathrm{j} \in \overline{1, \mathrm{~N}^{(2)}} \quad$ и $\quad$ весов $\mathrm{w}_{\mathrm{ltj}}^{(1)}(\mathrm{n}), \quad \mathrm{i} \in \overline{1, \mathrm{~N}^{(0)}}, \quad \mathrm{j} \in \overline{1, \mathrm{~N}^{(1)}}, \quad \mathrm{t} \in \overline{0, \mathrm{M}^{(0)}}$, $\mathrm{w}_{\mathrm{ij}}^{(2)}(\mathrm{n}), \quad \mathrm{i} \in \overline{1, \mathrm{~N}^{(1)}}, \quad \mathrm{j \in} \overline{1, \mathrm{~N}^{(2)}}$, где $\mathrm{N}^{(\mathrm{k})}$ количество нейронов в $k$-м слое, $M^{(0)}-$ задержка во входном слое.

2. Задается обучающее множество $\left\{\left(x_{\mu}, d_{\mu}\right) \mid x_{\mu} \in R^{N^{(0)}}, d_{\mu} \in R^{N^{(2)}}\right\}, \quad \mu \in \overline{1, P}$, где $x_{\mu}-\mu$-й обучающий входной вектор, $d_{\mu}-\mu$-й обучающий выходной вектор, $P-$ мощность обучающего множества.

3. $\mu=1$.

4. Начальная инициализация

$$
y_{\mu i}^{(0)}(n-v)=0, v \in \overline{1, M^{(0)}}, i \in \overline{1, N^{(0)}} \text {. }
$$

5. Вычисление выходного сигнала для первого слоя (прямой ход)

$$
\begin{gathered}
y_{\mu i}^{(0)}(n)=x_{\mu i}, i \in \overline{1, N^{(0)}}, \\
y_{\mu j}^{(1)}(n)=f^{(1)}\left(s_{\mu j}^{(1)}(n)\right), \quad j \in \overline{1, N^{(1)}},
\end{gathered}
$$


$s_{\mu j}^{(1)}(n)=\sum_{t=0}^{M^{(0)}} \sum_{i=0}^{(0)} w_{i t j}^{(1)}(n) y_{\mu i}^{(0)}(n-t)$,

Считается, что

$$
w_{0 j}^{(1)}(n)=b_{j}^{(1)}(n), y_{\mu 0}^{(1)}(n)=1 .
$$

6. Если $\mu<P$, то $\mu=\mu+1$, переход к пункту 5.

7. $\mu=1$.

8. Вычисление выходного сигнала для второго слоя (прямой ход)

$$
\begin{gathered}
y_{\mu j}^{(2)}(n)=\frac{f^{(2)}\left(s_{\mu j}^{(2)}(n)\right),}{j \in 1, N^{(2)}}, \\
s_{\mu j}^{(2)}(n)=\sum_{i=0}^{N^{(1)} w_{i j}^{(2)}(n) y_{\mu i}^{(1)}(n) .}
\end{gathered}
$$

Считается, что

$$
\begin{aligned}
& w_{0 j}^{(2)}(n)=b_{j}^{(2)}(n), \\
& y_{\mu 0}^{(1)}(n)=1 .
\end{aligned}
$$

9. Если $\mu<P$, то $\mu=\mu+1$, переход к пункту 8.

10. Вычисление энергии ошибки искусственной нейросети (ИНС)

$$
\begin{gathered}
E(n)=\frac{1}{2 P} \sum_{\mu=1}^{P} \sum_{j=1}^{N} e_{\mu j}^{(2)}(n), \\
e_{\mu j}(n)=y_{\mu j}^{(2)}(n)-d_{\mu j} .
\end{gathered}
$$

11. Настройка синаптических весов на основе обобщенного дельта-правила (обратный ход)

$$
\begin{aligned}
w_{i j}^{(2)}(n+1) & =w_{i j}^{(2)}(n)-\eta \frac{\partial E(n)}{\partial w_{i j}^{(2)}(n)}, \\
& i \in \overline{0, N^{(1)}}, \\
j & \in \overline{1, N^{(2)}}, \\
w_{l t j}^{(1)}(n+1) & =w_{l t j}^{(1)}(n)-\eta \frac{\partial E(n)}{\partial w_{l t j}^{(1)}(n)}, \\
i & \overline{0, N^{(0)}}, \\
j & \overline{1, N^{(1)}}, \\
t & \in \overline{0, M^{(0)}},
\end{aligned}
$$

$$
\begin{gathered}
\frac{\partial E(n)}{\partial w_{i j}^{(2)}(n)}=\frac{1}{P} \sum_{\mu=1}^{P} y_{\mu i}^{(1)}(n) g_{\mu j}^{(2)}(n), \\
\frac{\partial E(n)}{\partial w_{l t j}^{(1)}(n)}=\frac{1}{P} \sum_{\mu=1}^{P} y_{\mu i}^{(0)}(n-t) g_{\mu j}^{(1)}(n),
\end{gathered}
$$

$$
g_{\mu j}^{(2)}(n)=f^{\prime(2)}\left(s_{\mu j}^{(2)}(n)\right)\left(y_{\mu j}^{(2)}(n)-d_{\mu j}\right),
$$

$$
g_{\mu j}^{(1)}(n)=f^{\prime(1)}\left(s_{\mu j}^{(1)}(n)\right) \sum_{l=1}^{N^{(2)}} w_{j l}^{(2)}(n) g_{\mu l}^{(2)}(n) .
$$

12. Проверка условия завершения: если $E(n)<\varepsilon$, то завершение, иначе $-n=n+1$, переход к пункту 2.

При использовании видеокарты GeForce 920 М пакетный режим обучения предложенной авторской нейросети привел к увеличению скорости прямого хода приблизительно на $P\left(N^{(1)}+N^{(2)}\right)$ раз, а обратного - на $P$ раз.

Заключение. Имеющиеся сегодня методы для диагностики вентиляторной установки главного проветривания были исследованы с целью решения проблемы снижения вероятности возникновения ошибок диагностики.

Данные проведенных исследований позволили прийти к заключению о том, что искусственные нейронные сети на сегодня представляют собой наиболее эффективный метод диагностики.

С целью повышения качества диагностики была предложена авторская нейронная сеть.

В ходе исследования была установлена структура модели указанной авторской нейронной сети.

Результаты проведенных экспериментов продемонстрировали отсутствие существенных изменений значения среднеквадратичной ошибки при использовании более десяти скрытых нейронов. Предложенная сеть позволяет получить минимум возможных отклонений при диагностике.

Практическая значимость выражена в возможности увеличения скорости прямого хода приблизительно на $P\left(N^{(1)}+N^{(2)}\right)$ раз, а обратного - на $P$ раз, при использовании представленного пакетного режима обучения. 


\section{Список литературы}

1. Ширман А. Р., Соловьев А. Б. Практическая вибродиагностика и мониторинг состояния механического оборудования. Москва, 1996. $276 \mathrm{c.}$

2. Барков А. В., Баркова Н. А., Азовцев А. Ю. Мониторинг и диагностика роторных машин по вибрации. Санкт-Петербург: Изд. центр СПбГМТУ, 2000. 169 с.

3. Баркова В. А. Современное состояние виброакустической диагностики машин. Санкт-Петербург: Ассоциация ВАСТ, 2002. $100 \mathrm{c}$.

4. Гольдин А. С. Вибрация роторных машин. Москва: Машиностроение, 1999. 344 с.

5. Клюева В. В. Неразрушающий контроль и диагностика: справочник. Москва: Машиностроение, 2003. 300 c.

6. Федоров Е. Е. Методики интеллектуальной диагностики: монография. Донецк: Ноулидж, 2010. 303 c.

7. Бабак Г. А., Бочаров К. П., Волохов А. Т. Шахтные вентиляторные установки главного проветривания. Москва: Недра, 1982. 296 с.

8. Носырев Б. А., Белов С. В. Вентиляторные установки шахт и метрополитенов: учеб. пособ. Екатеринбург: Изд-во Уральской гос. горно-геолог. акад., 2000. 278 с.

9. Галушкин А. И. Теория нейронных сетей. Москва: ИПРЖР, 2000. 416 с.

10. Каллан Р. Основные концепции нейронных сетей. Москва: Вильямс, 2001. 288 с.

11.Sivanandam S. N., Sumathi S., Deepa S. N. Introduction to neural networks using Matlab 6.0. New Delhi: The McGraw-Hill Comp. Inc., 2006. $660 \mathrm{p}$.

12.Руденко О. Г., Бодянський С. В. Штучні нейронні мережі. Харків: Компанія СМIT, 2006. $404 \mathrm{c}$.

13.Галушкин А. И., Цыпкин Я. 3. Нейронные сети: история развития теории: учеб. пособ. для вузов. Москва: ИПРЖР, 2001. 840 с.

14.Осовский С. Нейронные сети для обработки информации. Москва: Финансы и статистика, 2002. 344 с.

15.Комарцова Л. Г., Максимов А. В. Нейрокомпьютеры. Москва: Изд-во МГТУ им. Н. Баумана, 2002. 320 c.

16. Haykin S. Neural networks. New York: Pearson Education, 1999. 823 p.

17.Федоров Е. Е. Искусственные нейронные сети: монография. Красноармейск: ДонНТУ, 2016. 338 c.

\section{References}

1. Shirman, A. R., Solov'ev, A. B. (1996) Practical vibration diagnostics and monitoring of mechanical equipment condition. Moscow, 276 p. [in Russian].

2. Barkov, A. V., Barkova, N. A., Azovtsev, A. Yu. (2000) Monitoring and diagnostics of rotary machines by vibration, St. Petersburg, 169 p. [in Russian].

3. Barkova, V. A. (2002) Current state of vibroacoustic machine diagnostics. St. Petersburg: Assotsiatsiya VAST, 100 p. [in Russian].

4. Gol'din, A. S. (1999) Vibration of rotary machines. Moscow: Mashinostroenie, 344 p. [in Russian].

5. Klyueva, V. V. (2003), Non-destructive testing and diagnostics: a handbook. Moscow: Mashinostroenie, 300 p. [in Russian].

6. Fedorov, E. E. (2010) Methods of intellectual diagnostics: monograph, Donetsk: Noulidzh, 303 p. [in Russian].

7. Babak, G. A., Bocharov, K. P., Volokhov, A. T. (1982) Mine fan installations of main ventilation, Moscow: Nedra, 296 p. [in Russian].

8. Nosyrev, B. A., Belov, S. V. (2000) Fan installations of mines and undergrounds: manual. Ekaterinburg: Izd-vo Ural'skoi gos. gorno-geolog. akad., 278 p. [in Russian].

9. Galushkin, A. I. (2000) Theory of neural networks. Moscow: IPRZhR, 416 p. [in Russian].

10. Kallan, R. (2001) Basic concepts of neural networks, Moscow: Vil'yams, 288 p. [in Russian].

11.Sivanandam, S. N., Sumathi, S., Deepa, S. N. (2006) Introduction to neural networks using Matlab 6.0. New Delhi: McGraw-Hill Comp. Inc., $660 \mathrm{p}$.

12.Rudenko, O. G., Bodjans'kyj Je. V. (2006) Artificial neural networks. Harkiv: Kompanija SMIT, 404 p. [in Ukrainian].

13.Galushkin, A. I., Cipkin Ja. Z. (2001) Neural networks: the history of theory development. Moscow: IPRZhR, 840 p. [in Russian].

14. Osovskyj, S. (2002) Neural networks for information processing, Moscow: Finansy i statistika, 344 p. [in Russian].

15. Komarcova, L. G., Maksimov, A. V. Neurocomputers. Moscow: Izd-vo MGTU im. N. Baumana, 320 p. [in Russian].

16. Haykin, S. (1999) Neural networks. New York: Pearson Education, 823 p.

17.Fedorov, E. E. (2016) Artificial neural networks: monograph. Krasnoarmejsk: DonNTU, 338 p. [in Russian]. 
E. E. Fedorov ${ }^{1}$, Dr.Tech.Sc., associate professor, professor, e-mail: fedorovee75@ukr.net

I. V. Yarosh' ${ }^{2}$, senior lecturer, e-mail: iryna.yarosh@donntu.edu.ua

T. A. Cherniak ${ }^{2}$, assistant,

e-mail: tetiana.cherniak@donntu.edu.ua

${ }^{1}$ Cherkasy State Technological University

Shevchenko blvd, 460, Cherkasy, 18006, Ukraine,

${ }^{2}$ Donetsk National Technical University

Shybankova square, 2, Pokrovsk, 85300, Ukraine

\section{METHOD OF BATCH TRAINING OF NEURAL NETWORK WITH DELAY IN INPUT LAYER FOR INTEGRATED DIAGNOSTICS OF THE STATE OF FAN INSTALLATION OF THE MAIN AIRING}

Currently, the increasing of operational safety is one of the major problems that exist in mining industry. The problem of mining equipment accident rate is caused by avalanche accumulating part of its physical resource exhaustion. Fan installation of the main airing, which ensures normal vital activities of mine personnel, is of the most importance among mining equipment. Therefore, an important task is to develop a software component, designed to diagnose the state of it and to be used in computer systems. The problem of building effective methods, providing high speed of diagnostics model training, as well as a high probability, adequacy and speed of recognition of signals, which contain the vibrational information, lies at the heart of this objective. At present, as a tool for vibration diagnostics, such calculation methods as: kurtosis, crest factor, RMS value, envelope spectrum are most commonly used. However, when using these markers separately for diagnosis of fan installations of the main airing condition, the probability of error is no less than 0.05. On the other hand, the processing speed of vibrational information is poor. Therefore, the development of methods for intelligent integrated diagnostics of fan installations of the main airing is relevant. As the use of artificial neural networks in the diagnosis gives a tangible advantage, which is that: the interaction between the factors is studied on finished models; it does not require any assumptions regarding the distribution of factors; a priori information about the factors can be omitted; the initial data can be highly correlated, incomplete or noisy; it is possible to conduct the analysis of systems with a high degree of nonlinearity; fast model development; high adaptability; the analysis of systems with a large number of factors; it does not require a complete enumeration of all possible models; the analysis of systems with non-uniform factors, neural network method of diagnosis is used in the article. The aim of the study is to develop a method for analysis of the process of changing the condition of fan installations of the main airing. The model structure of an artificial neural network, which is a multilayer perceptron with time delay in the input layer and provides a comprehensive analysis of diagnostic factors, is determined in the article. As a criterion for evaluation the efficiency of neural network model diagnostics the minimum of mean-square error is chosen. As a result of numerical study, it has been found that ten hidden neurons does not change the RMS error significantly, the proposed network provides the diagnostic results with minimum deviation. The use of the proposed batch training mode has made it possible to accelerate the forward stroke for approximately $P\left(N^{(1)}+N^{(2)}\right)$ times, and the reverse for approximately $P$ times, where $P$ is the power of training set, $N^{(k)}$ is the number of neurons in k-layer. The created algorithms can be used to solve problems associated with the diagnosis of electromechanical objects.

Keywords: integrated diagnostics, fan installation of the main airing, artificial neural network, process safety, batch training mode. 
Є. Є. Федоров ${ }^{1}$, д.т.н., доиент, професор,

e-mail: fedorovee75@ukr.net

I. В. Ярош², старший викладач,

e-mail: iryna.yarosh@donntu.edu.ua

Т. А. Черняк ${ }^{2}$, асистент,

e-mail: tetiana.cherniak@donntu.edu.ua

${ }^{1}$ Черкаський державний технологічний університет

бульв. Шевченка, 460, м. Черкаси, 18006, Україна

${ }^{2}$ Донецький національний технічний університет

пл. Шибанкова, 2, м. Покровськ, 85300, Україна

\section{МЕТОД ПАКЕТНОГО НАВЧАННЯ НЕЙРОМЕРЕЖІ \\ 3 ЗАТРИМКОЮ У ВХІДНОМУ ШАРІ ДЛЯ КОМПЛЕКСНОЇ ДІАГНОСТИКИ СТАНУ ВЕНТИЛЯТОРНОЇ УСТАНОВКИ ГОЛОВНОГО ПРОВІТРЮВАННЯ}

У статті розглянуто і представлено дані виконаного аналізу наявних методів для здійснення діагностики стану вентиляційної установки головного провітрювання на гірничопромисловому підприємстві (шахті). Виконані дослідження спрямовані на зниження ймовірності виникнення помилок діагностики. Переваги і недоліки зазначених існуючих методів враховані при розробиі та реалізаиії нейромережевого методу діагностики вентиляційної установки головного провітрювання. Проведені експерименти дозволили створити архітектуру нейронної мережі з певною затримкою у вхідному шарі, яка послужила основою для розробки наведеного методу. Можливість прискореного навчання надається за рахунок запропонованого в роботі пакетного режиму. Ефективність методу було оцінено в результаті численних досліджень, які відображають прачездатність мережі й архітектури. Запропонована в роботі нейронна мережа дозволяе отримати мінімум відхилень при діагностиці.

Ключові слова: комплексна діагностика, вентиляторна установка головного провітрювання, штучна нейронна мережа, виробнича безпека, пакетний режим навчання. 